\title{
Causes of Failure after Total Knee Arthroplasty in Osteoarthritis Patients 55 Years of Age or Younger
}

\author{
Kyung Tae Kim, MD, Song Lee, MD, Dong Oh Ko, MD, Bong Soo Seo, MD, Woo Shik Jung, MD, and \\ Byung Kwon Chang, MD \\ Department of Orthopedic Surgery, Seoul Sacred Heart General Hospital, Seoul, Korea
}

Purpose: To identify the modes of failure after total knee arthroplasty (TKA) in patients $\leq 55$ years of age and to compare with those $>55$ years of age in patients who underwent revision TKA.

Materials and Methods: We retrospectively reviewed 256 revision TKAs among patients who underwent TKA for knee osteoarthritis between January 1992 and December 2012. The causes of TKA failure were analyzed and compared between the groups.

Results: Thirty-one revision TKAs were performed in patients $\leq 55$ years of age at the time of primary TKA, whereas 225 cases were in those $>55$ years of age at primary TKA. In the $\leq 55$ years of age group, the most common cause of TKA failure was polyethylene wear (45\%) followed by infection (26\%) and loosening (17\%). The interval from primary TKA to revision was 8.6 years (range, 1 to 17 years). There were relatively lower infection rate and higher loosening rate in patients $\leq 55$ years of age, but the difference was not statistically significant.

Conclusions: The main causes of failure after TKA in patients $\leq 55$ years of age were polyethylene wear, infection and loosening, and there was no significant difference in the modes of failure after TKA between the two groups.

Keywords: Knee, Arthroplasty, Failure, Revision, Younger age

\section{Introduction}

Total knee arthroplasty (TKA) has been established as the treatment of choice for advanced degenerative arthritis. It has demonstrated excellent clinical efficacy in terms of postoperative pain, physical function, and patient satisfaction and contributed to long-term implant survival and patient's quality of life ${ }^{1,2)}$. The procedure has been performed commonly on elderly patients and rarely on young patients with knee joint disorders accompanied by severe pain, such as rheumatoid arthritis ${ }^{3,4}$. Recently, the

Received November 5, 2013; Revised January 9, 2014;

Accepted January 14, 2014

Correspondence to: Kyung Tae Kim, MD

Department of Orthopedic Surgery, Seoul Sacred Heart General

Hospital, 259 Wangsan-ro, Dongdaemun-gu, Seoul 130-011, Korea

Tel: +82-2-966-1616, Fax: +82-2-968-2394

E-mail: kktkimos@hanmail.net

This is an Open Access article distributed under the terms of the Creative Commons Attribution Non-Commercial License (http://creativecommons.org/licenses/by-nc/3.0/) which permits unrestricted non-commercial use, distribution, and reproduction in any medium, provided the original work is properly cited. number of TKAs among young patients has been increasing. It is attributable to the growing incidence and severity of degenerative arthritis in young patients and the expansion of indications of the procedure due to improved implant survival rates and achievement of favorable outcomes from young arthritis patients ${ }^{2,5}$. In general, TKA in young patients has been associated with relatively unsatisfactory results and different causes of failure from those in elderly patients ${ }^{4,6}$. However, most of the research on TKA in young patients involved those with rheumatoid arthritis; thus, it is difficult to find studies that enrolled those with degenerative arthritis alone. In particular, there is scant literature available regarding the relationship between age and the cause of failure after TKA. In this study, we investigated causes of failure of TKA in patients 55 years of age or younger and made comparisons with patients older than 55 years of age among those who underwent revision TKA.

\section{Materials and Methods}

Causes of failure were investigated in 240 patients (256 cases) who had revision surgery after primary TKA that had been 


\section{Kim et al. Failure after Total Knee Arhtroplasty in Younger Patients}

performed under the diagnosis of degenerative arthritis at our institution between January 1992 and December 2012 and were compared between those $\leq 55$ years of age and those $>55$ years of age. The age at the time of primary surgery was $\leq 55$ years in 30 patients ( 31 knees) and $>55$ years in 210 patients ( 225 knees).

Clinical and radiographic follow-up examinations were performed 3 months, 6 months, and 12 months after primary TKA and yearly afterwards. Knees that were revised due to TKA failure during the follow-up were reviewed retrospectively. The modes of failure identified through clinical and radiographic assessments and intraoperative observation were classified according to the standardized list and definition of the Knee Society ${ }^{7}$.

Statistical analysis was done using the Fisher exact probability test, with $\mathrm{p}<0.05$ considered statistically significant.

\section{Results}

A total of 11,816 TKAs were performed at our institution between 1992 and 2012. Of these, revision was required in 256 cases

Table 1. Patient Demographics

\begin{tabular}{lccc}
\hline \multicolumn{1}{c}{ Variable } & $\leq 55$-yr-old & $>55$-yr-old & Total \\
\hline Total TKA & 453 & 11,363 & 11,816 \\
Failure & $31(7)$ & $225(2)$ & $256(2.2)$ \\
Gender (M/F) & $3 / 27$ & $23 / 187$ & $26 / 214$ \\
Age (yr) at primary TKA & $50.6 \pm 5.85$ & $65.7 \pm 5.98$ & $63.9 \pm 7.39$ \\
Age (yr) at revision TKA & $59.2 \pm 8.22$ & $70.5 \pm 6.81$ & $69.5 \pm 7.94$ \\
Time to failure (yr) & $8.6 \pm 4.30$ & $5.3 \pm 3.97$ & $5.7 \pm 4.18$ \\
\hline
\end{tabular}

Values are presented as number (\%) or mean \pm standard deviation.

TKA: total knee arthroplasty.
(240 patients, 2.2\%). Of the 240 patients with revision TKA, 26 were male and 214 were female. The surgery was unilateral in 224 patients and bilateral in the remaining 16 patients. Their mean age was 63.9 years (range, 40 to 80 years) at the time of primary TKA and 69.5 years (range, 41 to 85 years) at revision surgery. There was no significant difference in gender, the interval between the operations, and the initial implant between the $\leq 55$ years of age group and the $>55$ years of age group $(\mathrm{p}>0.05)$ (Table 1$)$.

The major cause of TKA failure was polyethylene wear in 113 cases $(44.1 \%)$, infection in 99 cases $(38.7 \%)$, and component loosening in 31 cases (12.1\%). The mean interval between the primary TKA and the revision surgery was 5.7 years (range, 0.1 to 18 years) (Table 2).

\section{TKA Failure in Patients $\leq 55$ Years of Age}

A total of 453 TKAs were performed in $\leq 55$-year-old patients between 1992 and 2012. Of these, 31 cases (7\%) were revised at our institution. Their mean age was 50.6 years (range, 40 to 55 years) at primary surgery and 59.2 years (range, 41 to 70 years) at revision surgery (Table 1).

1) Type of implant for primary TKA

The type of implant used in primary TKA was Maxim (Biomet, Warsaw, IN, USA) in 169 cases, Scorpio (Stryker Orthopaedics, Mahwah, NJ, USA) in 98 cases, Vanguard (Biomet) in 59 cases, Nexgen (Zimmer Inc., Warsaw, IN, USA) in 56 cases, Advance (Wright Medical Technology Inc., Memphis, TN, USA) in 51 cases, and Sigma (DePuy Orthopaedics Inc., Warsaw, IN, USA) in 20 cases. In particular, the implant that was used in the 31 revised knees was Maxim in 12 cases, Scorpio in 16 cases, and Nexgen in 3 cases.

Table 2. Comparisons of Failure Modes after Total Knee Arthroplasty between $\leq 55$ Years of Age Group and >55 Years of Age Group

\begin{tabular}{|c|c|c|c|c|c|c|c|}
\hline \multirow{2}{*}{ Mode of failure } & \multicolumn{2}{|c|}{$\leq 55$-yr-old } & \multicolumn{2}{|c|}{$>55$-yr-old } & \multicolumn{2}{|c|}{ Total } & \multirow{2}{*}{$\mathrm{p}$-value } \\
\hline & No. (\%) & Time (yr) & No. (\%) & Time (yr) & No. (\%) & Time $(y r)$ & \\
\hline Wear & $14(45.2)$ & 10.5 & $99(44.0)$ & 7.8 & $113(44.1)$ & 8.1 & 1.00 \\
\hline Infection & $8(25.8)$ & 5.6 & $91(40.4)$ & 2.2 & $99(38.7)$ & 2.3 & 0.17 \\
\hline Loosening & $5(16.2)$ & 9 & $26(11.6)$ & 6.7 & $31(12.1)$ & 7.1 & 0.55 \\
\hline Stiffness & $1(3.2)$ & 7 & $1(0.4)$ & 1 & $2(0.8)$ & 1 & 0.23 \\
\hline Periprosthetic fracture & $1(3.2)$ & 8 & $2(0.9)$ & 4 & $3(1.1)$ & 3.7 & 0.32 \\
\hline Malalignment & $1(3.2)$ & 1 & $0(0)$ & 0 & $1(0.4)$ & 1 & 0.12 \\
\hline Osteolysis & $1(3.2)$ & 13 & $1(0.4)$ & 9 & $2(0.8)$ & 11 & 0.23 \\
\hline Instability & $0(0)$ & 0 & $5(2.3)$ & 4 & $5(2.0)$ & 2.3 & 1.00 \\
\hline Total & $31(100)$ & 8.6 & $225(100)$ & 5.3 & $256(100)$ & 5.7 & \\
\hline
\end{tabular}

${ }^{\text {a) }}$ Fisher exact probability test. 
2) Cause of failure

The major causes of failure include polyethylene wear in 14 cases (45\%), infection in 8 cases (26\%), and component loosening in 5 cases (17\%). The followings were associated with failure in one case each (3\%): stiffness, periprosthetic fracture, malalignment, and osteolysis (Table 2, Figs. 1 and 2).

\section{3) Interval between primary TKA and revision}

The mean interval between the operations was 8.6 years (range, 1 to 17 years) for the 31 cases: the mean period was 5.6 years (range, 2 to 10 years) for infected knees, 9 years (range, 3 to 13 years) for knees with component loosening, and 10.5 years ( 2 to 17 years) for those with polyethylene wear (Table 2).

\section{4) Revision TKA}

Two-stage revision TKA was performed for infected knees, whereas one-stage revision was done in the remaining knees. The prosthesis of choice for revision was Scorpio (Stryker) in 17 cases, Maxim (Biomet) in 11 cases, and Nexgen (Zimmer Inc.) in
3 cases.

\section{TKA Failure in Patients $>55$ Years of Age}

Of the 11,363 TKAs that were performed in $>55$-year-old patients, $225(2 \%)$ required a revision. The mean interval between the operations was 5.3 years (range, 0.1 to 18 years). The major causes of failure of primary TKA include polyethylene wear in 99 cases (44\%), infection in 91 cases (40\%), and component loosening in 26 cases (12\%). The other conditions led to TKA failure were instability in 5 cases $(2.3 \%)$, periprosthetic fracture in 2 cases $(0.9 \%)$, stiffness in 1 case $(0.4 \%)$, and osteolysis in 1 case (0.4\%) (Table 2).

\section{Intergroup Comparisons}

In both groups, the most common cause of failure was polyethylene wear, which was followed by infection and component loosening. Although the infection rate was lower and the incidence of component loosening was higher in the $\leq 55$ years of age group, no statistically significant difference was found between
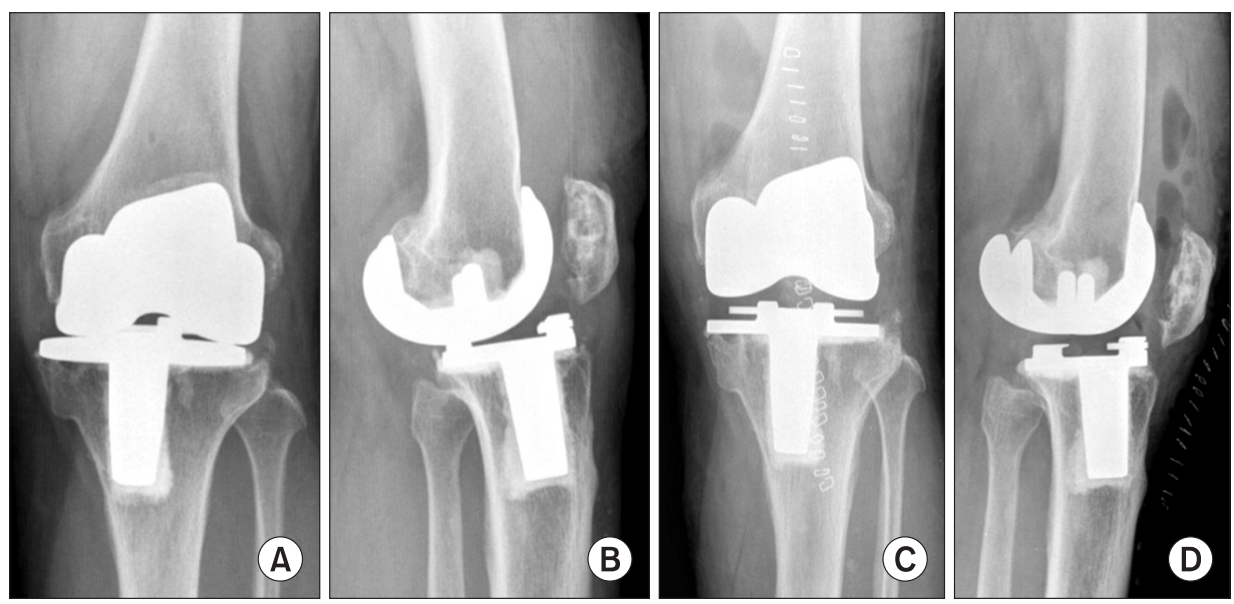

Fig. 1. (A, B) Radiographs showing failure of total knee arthroplasty (TKA) that occurred 11 years after primay TKA due to polyethlylene wear in a 50-year-old female. (C, D) Radiographs obtained after revision TKA for polyethylene insert change.
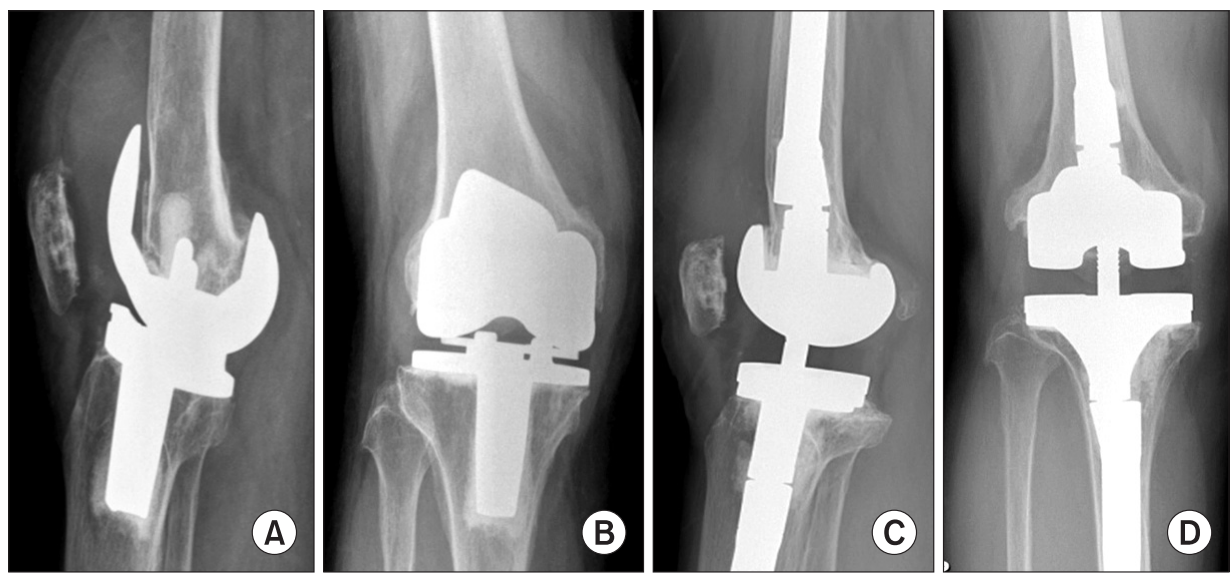

Fig. 2. (A, B) Radiographs showing failure of total knee arthroplasty (TKA) that occurred 11 years after primay TKA due to aseptic loosening in a 53-year-old female. (C, D) Radiographs obtained after revision TKA using a metal block and a long stem. 


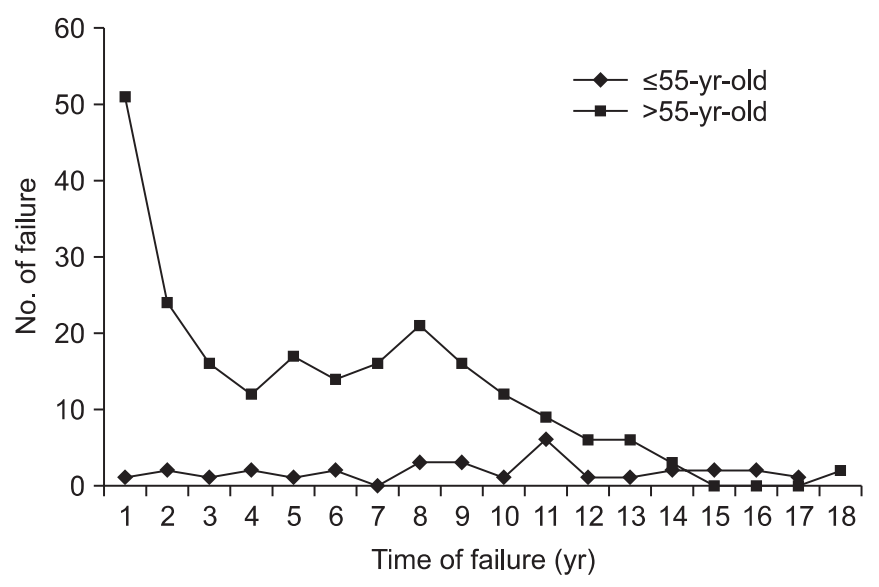

Fig. 3. The graph shows the frequency of failure over the time after total knee arthroplasty. In the $>55$ years of age group, the incidence of failure was the highest in the first 2 postoperative years and then declined continuously after total knee arthroplasty. The incidence of failure was relatively constant over the time after total knee arthroplasty in the $\leq 55$ years of age group.

the groups $(\mathrm{p}>0.05)$. The mean interval between the operations was shorter in the $>55$ years of age group ( 5.3 years; range, 0.1 to 18 years) than in the younger patient group ( 8.6 years; range, 1 to 17 years), but there was no notable intergroup difference ( $p>0.05)$. The frequency of failure was high within the first 2 postoperative years and gradually decreased afterwards in the $>55$ years of age group, whereas the value was relatively constant in the younger group (Fig. 3). Infection-related failure occurred at a mean of 5.6 years (range, 2 to 10 years) after primary TKA in the $\leq 55$ years of age group and 2.2 years (range, 0.1 to 10 years) after primary TKA in the other group. The mean period between primary TKA and component loosening was 9 years (range, 3 to 13 years) in the younger group and 6.7 years (range, 2 to 18 years) in the other group. Polyethylene wear was observed at a mean of 10.5 years (range, 2 to 17 years) after primary TKA in the younger group and 7.8 years (range, 0.2 to 18 years) after primary TKA in the other group (Table 2).

\section{Discussion}

The significance of the study resides in the analysis of the modes of TKA failure in a relatively large number of young patients $\leq 55$ years of age with degenerative arthritis who were enrolled at a single institution. TKA has exhibited excellent clinical outcomes and improved implant durability and quality of life; thus, the frequency of TKA has been on the rise constantly. High long-term implant survival rates have resulted in increasing demand for the treatment among younger patients and rising patient expectations. In addition, due to the surgeon's confidence in the surgical procedure based on the experience of obtaining favorable outcomes in young patients with rheumatoid arthritis, TKA has become considered as a treatment of choice in young patients with degenerative arthritis. However, the results of TKAs were less satisfactory in younger patients and recent research using the social, academic, and national registry data showed that revision rates were higher in younger patients ${ }^{4,6)}$.

In general, the 10-year survival rate of TKA is considered $90 \%-98 \%$ and some studies even reported the 15 to 20 -year survival rate was as high as $96 \%{ }^{4,6,8)}$. In the meta-analysis of 13 studies, Keeney et al. ${ }^{6)}$ documented the initial 6 to 10 -year survival rates were calculated as $90.6 \%-99 \%$ and the $\geq 15$-year survival rates as $85 \%-96.5 \%$ in $<55$ years of age patients. Heyse et al. ${ }^{4)}$ reported that the 10-year survivorship of TKA was $95 \%$ and the 20-year survivorship was $82 \%$ in patients with juvenile idiopathic arthritis (mean age, 30 years; range, 11 to 58 years). Julin et al. ${ }^{9)}$ analyzed Finnish Arthroplasty Register and found that the early failure rate after TKA was high in $\leq 55$ years of age patients: the 5 -year survival rate was $92 \%$ in the $\leq 55$ years of age group, $95 \%$ in the 56 to 65 years of age group, and $97 \%$ in the $>65$ years of age group. Vessely et al. ${ }^{8)}$ reported that the 15 -year survival rate was significantly low in the less than 60 -year-old group compared to the 60 to 80 -year-old group and the more than 80 -year-old group (82.6\%, 95.1\%, and 99.0\%, respectively) based on the assessment of 1,000 cases of TKA. These high failure rates in young patients compared to those in elderly patients may be associated with the higher level of activities and functional demands, longer remaining life expectancy, and the greater prevalence of obesity in young patients ${ }^{5,9}$. Heyse et al. ${ }^{4)}$ suggested that the survivorship of TKA in patients with juvenile idiopathic arthritis may be affected by poor bone quality, contractures, and immunosuppressive medications. Odland et al. ${ }^{10)}$ stated that there would be difference in the outcomes of TKA between rheumatoid arthritis patients and degenerative arthritis patients. On the other hand, Bisschop et al. ${ }^{1)}$ reported that the survival rates and function scores were not significantly different between the rheumatoid arthritis patients and degenerative arthritis patients in $\leq 60$ years of age cohorts. In our study, the TKA failure rate was $7 \%$ in the $\leq 55$ years of age group and $2 \%$ in the $>55$ years of age group. However, we acknowledge that the study results were based only on the retrospective review of patients who underwent both the primary and revision TKAs at our institution and the failure to conduct follow-up of the total TKA cases (11,816 knees) may have compromised the accuracy of the survival rate calculation.

The common causes of failure after TKA include infection, 
polyethylene wear, instability, aseptic loosening, extensor mechanism problems, aseptic necrosis of the patella, periprosthetic fracture, and arthrofibrosis ${ }^{11)}$. Sharkey et al. ${ }^{12)}$ reviewed 212 TKAs and noted that most of the failures were due to polyethylene wear (25\%), aseptic loosening (24.1\%), instability (21.2\%), and infection (17.5\%). Fehring et al. ${ }^{13)}$ reported that revision surgery was necessitated in 279 of the 440 knees (63\%) within 5 years after primary TKA because of infection (38\%), instability (27\%), bone ingrowth failure (8\%), patellofemoral problems (8\%), and implant wear or osteolysis (7\%). Bae et al. ${ }^{14)}$ identified the most common cause of failure as polyethylene wear, which was followed by deep infection and aseptic loosening, whereas according to Cho et al. ${ }^{15)}$, the leading causes of failure, in order of frequency, were infection, loosening, and polyethylene wear. In 2013, Kasahara et al. ${ }^{16)}$ reported that revision was required in $3.3 \%$ of the 4,047 TKAs that were performed in 5 different institutions and the most common cause of failure was mechanical loosening (40\%) followed by infection (24\%), wear/osteolysis (9\%), and instability (9\%). The results of our study are similar to those of the above-mentioned studies: in our study, $44.1 \%$ had revision for polyethylene wear, $38.7 \%$ for infection, and $12.1 \%$ for component loosening.

Most TKA failures are caused by mechanical overload due to malalignment or overload related to patient activities. Joint instability due to increased implant loads eventually leads to failure of total knee implants ${ }^{17}$. Bisschop et al. ${ }^{1)}$ claimed that younger patients are exposed to higher risk of prosthetic loosening, polyethylene wear, and component breakage compared to elderly patients due to their high level of physical activities that impose high mechanical loads on the implants. D'Lima et al. ${ }^{18)}$ suggested that knee forces affect component survivorship, wear of articular surfaces, and integrity of the bone-implant interface; therefore, excessive knee forces may result in the breakdown of the cement interface or collapse of underlying bone. Mechanically related failures are common in young patients after TKA, such as patellofemoral problems, implant breakage, wear, osteolysis, and loosening. Vessely et al. ${ }^{8)}$ investigated TKA failure patterns: mechanical failures defined as tibial component wear, tibio-femoral instability, aseptic loosening, or component fracture were not observed in any of the $>80$-year-old patients, whereas the 15 -year survivorship was $88.2 \%$ due to mechanical failures in $<60$-yearold patients. Heyse et al. ${ }^{4)}$ examined 349 knees in patients with juvenile idiopathic arthritis (mean age, 30 years; range, 11 to 58 years) for a mean period of 12 years (range, 2 to 33 years) after TKA: revision was required in 31 knees (8.9\%) primarily due to polyethylene failure or loosening (18 knees, $58 \%$ ) and due to infection (4 knees), stiffness ( 3 knees), periprosthetic fractures (2 knees), bilateral amputation for vascular reasons (2 knees), patellar resurfacing ( $1 \mathrm{knee}$ ), and instability ( $1 \mathrm{knee})$. Odland et al. ${ }^{10)}$ followed 67 TKAs that were performed in $\leq 55$ years of age patients with degenerative arthritis for a minimum of 10 years. In the study, aseptic loosening and/or osteolysis were the major causes of failure (11 cases, 16.4\%) followed by hematogenous infection ( 2 cases), instability ( 2 cases), and patellar fracture (1 case). In the analysis of Finnish Arthroplasty Register by Julin et al. ${ }^{9}$, infection was the cause of revision in a relatively small number of patients in the $\leq 55$-year-old patient group (19\%) compared to the 56 to 65 -year-old patient group (16\%) and $>65$-yearold patient group (26\%). Therefore, they concluded that the high rates of early TKA failure in young patients are associated with noninfectious reasons. In the current study, polyethylene wear (45\%) was most common in $\leq 55$-year-old patients with revision TKA, and infection (26\%) and component loosening (17\%) were also the major causes of failure in that age group. Other reasons for revision included stiffness, fracture, malalignment, and osteolysis. Compared to the $>55$ years of age group, polyethylene wear, infection, and component loosening were most prevalent in the revised knees in the $\leq 55$ years of age group as well. Although the younger patient group exhibited a lower infection rate and a higher incidence of component loosening, the intergroup differences were statistically insignificant.

Lee et al. ${ }^{19)}$ reported that infection-related failure of TKA occurred at a mean of 20.3 months postoperatively, whereas noninfectious failure developed at a mean of 221.5 months postoperatively. Koh et al. ${ }^{20)}$ suggested the incidence of failure was related to the patient's age. In our study, the mean interval from primary TKA to failure was shorter in the $>55$ years of age group (mean 5.3 years; range, 0.1 to 18 years) than in the $\leq 55$ years of age group (mean 8.6 years; range, 1 to 17 years), but no statistically significant difference was found between the groups. The incidence of failure was the highest within the first 2 postoperative years and gradually decreased afterwards in the $>55$ years of age group, whereas it was relatively constant in the other group. We attributed this to the relatively high rate of early failure caused by infection in the $>55$ years of age group. Polyethylene wear and implant loosening that could be considered as mechanical failures developed at a mean of 10.5 years and 9 years after primary TKA, respectively, in the $\leq 55$ years of age group, which was later when compared to 7.8 years and 6.7 years after surgery, respectively, in the $>55$ years of age group. The results were opposite of those shown in other studies; however, the intergroup difference was statistically insignificant, and thus survival rates should fur- 


\section{Kim et al. Failure after Total Knee Arhtroplasty in Younger Patients}

ther be investigated in a more precise manner ${ }^{1,8)}$.

TKA in young patients are expected to provide functional improvement and increased implant longevity ${ }^{21)}$. Functional improvement after TKA can be achieved by enhanced range of motion, increased motor performance and close to normal kinematic pattern. Implant longevity can be increased by more durable fixation, improvements in bearing materials, and lower polyethylene stresses ${ }^{3,21}$. Sharkey et al. ${ }^{12)}$ reported that improvement in implant design and material would contribute to reduced incidence of failure after TKA, and efforts should be made to prevent failures related to surgical techniques and iatrogenic failures. They suggested avoiding implants associated with accelerated polyethylene wear and osteolysis, use of improved materials, such as cross-linking polyethylene, and performance of precise surgical techniques to avert postoperative complications, such as instability and component malalignment or malposition. According to Dennis ${ }^{21}$, the use of mobile-bearing TKA designs should be considered as an option to reduce polyethylene wear by improving implant conformity without increasing loads transmitted to the fixation interface and reducing contact stresses. Some studies suggested that cementless fixation in TKA for young patients would allow for easy revision surgery in case of failure and lengthen the implant survivorship. However, the efficacy of cementless fixation has yet to be established, and it resulted in higher incidences of early loosening than did cemented fixation according to some studies ${ }^{12,13)}$.

One of the limitations of the study is that it is a retrospective review of revision TKAs that were performed at our institution only. In addition, the influence of surgical material used in primary TKA was not taken into consideration, and TKA survival rates could not be analyzed due to the possibility of patients having revisions at other institutions. In addition, the accuracy of statistical analysis could have been compromised due to the notable difference in the total number of cases between the groups. However, the significance of this study lies in the fact that a number of TAK revisions performed at a single institution was analyzed, a relatively great number of $\leq 55$ years of age patients with degenerative arthritis, not rheumatoid arthritis, were the subjects of the study.

\section{Conclusions}

The leading cause of failure after TKA in young patients $\leq 55$ years of age was polyethylene wear, followed by infection and component loosening. Although the infection rate was lower and the incidence of component loosening was higher in the younger patients when compared to patients $>55$ years of age, the differences did not demonstrate statistical significance.

\section{Conflict of Interest}

No potential conflict of interest relevant to this article was reported.

\section{References}

1. Bisschop R, Brouwer RW, Van Raay JJ. Total knee arthroplasty in younger patients: a 13-year follow-up study. Orthopedics. 2010;33:876.

2. Wood AM, McIlwain L, Arthur CH, Aitken SA, Keenan ACM, Walmsley P, Brenkel I. PFC sigma cobalt-chrome total knee replacement: early outcomes demonstrate no significant early failures at the three-year mark. Open J Orthopedics. 2014;4:1-6.

3. Engh GA. Advances in knee arthroplasty for younger patients: traditional knee arthroplasty is prologue, the future for knee arthroplasty is prescient. Orthopedics. 2007;30(8 Suppl):55-7.

4. Heyse TJ, Ries MD, Bellemans J, Goodman SB, Scott RD, Wright TM, Lipman JD, Schwarzkopf R, Figgie MP. Total knee arthroplasty in patients with juvenile idiopathic arthritis. Clin Orthop Relat Res. 2014;472:147-54.

5. W-Dahl A, Robertsson O, Lidgren L. Surgery for knee osteoarthritis in younger patients. Acta Orthop. 2010;81:161-4.

6. Keeney JA, Eunice S, Pashos G, Wright RW, Clohisy JC. What is the evidence for total knee arthroplasty in young patients?: a systematic review of the literature. Clin Orthop Relat Res. 2011;469:574-83.

7. Healy WL, Della Valle CJ, Iorio R, Berend KR, Cushner FD, Dalury DF, Lonner JH. Complications of total knee arthroplasty: standardized list and definitions of the Knee Society. Clin Orthop Relat Res. 2013;471:215-20.

8. Vessely MB, Whaley AL, Harmsen WS, Schleck CD, Berry DJ. The Chitranjan Ranawat Award: long-term survivorship and failure modes of 1000 cemented condylar total knee arthroplasties. Clin Orthop Relat Res. 2006;452:28-34.

9. Julin J, Jamsen E, Puolakka T, Konttinen YT, Moilanen T. Younger age increases the risk of early prosthesis failure following primary total knee replacement for osteoarthritis. a follow-up study of 32,019 total knee replacements in the Finnish Arthroplasty Register. Acta Orthop. 2010;81:413-9.

10. Odland AN, Callaghan JJ, Liu SS, Wells CW. Wear and lysis 
is the problem in modular TKA in the young OA patient at 10 years. Clin Orthop Relat Res. 2011;469:41-7.

11. Austin MS, Sharkey PF, Hozack WJ, Rothman RH. Knee failure mechanisms after total knee arthroplasty. Tech Knee Surg. 2004;3:55-9.

12. Sharkey PF, Hozack WJ, Rothman RH, Shastri S, Jacoby SM. Insall Award paper: why are total knee arthroplasties failing today? Clin Orthop Relat Res. 2002;(404):7-13.

13. Fehring TK, Odum S, Griffin WL, Mason JB, Nadaud M. Early failures in total knee arthroplasty. Clin Orthop Relat Res. 2001;(392):315-8.

14. Bae DK, Yoon KH, Kim HS, Song SJ, Yi JW, Kim YC. The results of revision total knee arthroplasty. J Korean Orthop Assoc. 2003;38:689-94.

15. Cho WS, Youm YS, Yang BS. The causes of revision total knee arthroplasty. J Korean Orthop Assoc. 2007;42:216-20.

16. Kasahara Y, Majima T, Kimura S, Nishiike O, Uchida J. What are the causes of revision total knee arthroplasty in Japan? Clin Orthop Relat Res. 2013;471:1533-8.
17. McTighe T, Clarke I. Failure mechanism on total knee arthroplasty [Internet]. Chagrin Falls, OH: Joint Implant Surgery Surgery \& Research Foundation; 2009 [cite $2014 \mathrm{Feb} 9$ ]. Available from: http://www.jisrf.org/.

18. D'Lima DD, Steklov N, Patil S, Colwell CW Jr. The Mark Coventry Award: in vivo knee forces during recreation and exercise after knee arthroplasty. Clin Orthop Relat Res. 2008;466:2605-11.

19. Lee KJ, Moon JY, Song EK, Lim HA, Seon JK. Minimum two-year results of revision total knee arthroplasty following infectious or non-infectious causes. Knee Surg Relat Res. 2012;24:227-34.

20. Koh IJ, Cho WS, Choi NY, Kim TK; Kleos Korea Research Group. Causes, risk factors, and trends in failures after TKA in Korea over the past 5 years: a multicenter study. Clin Orthop Relat Res. 2014;472:316-26.

21. Dennis DA. Trends in total knee arthroplasty. Orthopedics. 2006;29(9 Suppl):S13-6. 